\title{
An overview of thrombolytic therapy for pulmonary embolism: a single centre experience
}

\author{
NIKOLINA MARIĆ, MAJA MAČKOVIĆ, NIKOLA UDILJAK, DINKO BEKIĆ \\ Department of Emergency and Intensive Care Medicine, Clinic of Internal Medicine, University Hospital Sveti Duh, Zagreb
}

Corresponding author:

Nikolina Marić

Clinic of Internal Medicine, University Hospital Sveti Duh

Sveti Duh 64, Zagreb

Croatia

Phone: $+38513712-235$

fax: $+38513712-233$

E-mail:nikolinamaric1505@gmail.com

\section{ABSTRACT}

Pulmonary embolism (PE) is considered to be a major cause of mortality, morbidity and hospitalization in Europe. Haemodynamic benefits of thrombolysis in patients with shock and hypotension are undeniable, but the role of thrombolytic therapy on the outcome of haemodynamically stable patients still remains controversial.

This is a retrospective analysis of patients with acute PE treated with thrombolytic therapy in medical intensive care unit (ICU), University Hospital Sveti Duh, between March 2014 and April 2015.

Twenty two of 75 (29\%) patients with PE received thrombolytic therapy. The mean age of patients was 63 years, $45 \%$ were male and $55 \%$ female. The major symptoms were: dyspnea (73\%), chest pain (18\%) and syncope (9\%). $27 \%$ of patients receiving thrombolytic therapy were haemodynamically unstable and $73 \%$ were stable. All patients had an extensive clot burden on computed tomographic pulmonary angiography (CTPA). All haemodynamically stable patients had echocardiographic signs of right ventricular (RV) dysfunction. Troponin I was positive in all haemodynamically unstable patients and in $50 \%$ of haemodynamically stable patients. Only one (5\%) haemodynamically unstable patient died but not because of PE or therapy complication. All other patients survived and recovered completely. Two patients $(9 \%)$ had major non-intracranial bleeding complications, which were successfully treated with supportive therapy.

Key words: pulmonary embolism, thrombolytic therapy, right ventricular dysfunction, bleeding

\section{INTRODUCTION}

Acute pulmonary embolism (PE) is the most serious clinical presentation of venous thromboembolism (VTE). Overall annual incidence of VTE is 100-200 per 100000 inhabitants, and it is the third most frequent cardiovascular disease. $\mathrm{PE}$ is considered a major cause of mortality, morbidity and hospitalization in Europe. $(1,2)$ VTE can be a consequence of temporary or reversible risk factors such as surgery, trauma, immobilization, pregnancy, oral contraceptive use or hormone replacement therapy. VTE can also be 'unprovoked' if none of the known risk factors are present. Antithrombotic prophylaxis significantly reduces the risk of perioperative VTE. PE may also occur in the absence of any known risk factor. (3)

Clinical signs and symptoms of PE are non-specific. In most cases $\mathrm{PE}$ is suspected on the basis of dyspnoea, chest pain, presyncope or syncope and haemoptysis. (4) Based on the clinical status at presentation, patients are classified in two PE-related early mortality risk groups; high-risk being suspected or confirmed in the presence of shock or persistent hypotension and not high-risk in their absence. Risk stratification based on haemodynamic status is the first step in decision making strategies for both the diagnostic and therapeutic procedures. (5)

If $\mathrm{PE}$ is suspected in patient with shock or hypotension and computed tomographic pulmonary angiography (CTPA) is available, it should be done immediately. If CTPA is not available echocardiography is an alternative diagnostic test. In the case $\mathrm{PE}$ is suspected in a patient without shock or hypotension, the first step is to assess clinical probability of PE (Wells or Geneva score). If there is high clinical probability CTPA should be done. In the case of low clinical probability first D-dimer should be done and only if it is positive CTPA should be performed. $(4,5)$

Haemodynamic benefits of thrombolysis in PE patients with shock and hypotension are undeniable. Thrombolysis restores pulmonary perfusion more rapidly than anticoagulation with unfractionated heparin (UFH) alone. The early resolution of pulmonary obstruction leads to a prompt reduction in pulmonary artery pressure and resistance, with a concomitant improvement in right ventricular (RV) function. (6) The greatest benefit is observed when treatment is initiated within 48 hours of symptom onset, but thrombolysis can still be useful in patients who have had symptoms for 6-14 days. (7)

The effect of thrombolytic therapy on the outcome of haemodynamically stable patients who have submassive PE has been debated for years and remains controversial. $(8,9)$ The ongoing controversy is a result of several factors: the lack of large randomized study, the risk of serious bleeding associated with trombolytic therapy and the fact that heparin therapy alone can improve haemodynamic status gradually. (10)

Thrombolytic treatment carries a risk of major bleeding, including intracranial haemorrhage. (11) In the category of haemodynamically stable patients both the risk of bleeding and the benefits of accelerated lysis of clot must be taken into account. Decision making process on therapeutic strategy in patients with PE should 
be done promptly. It should be individualized and both benefits and risks should be carefully weighed on a case-by-case basis. Patient preferences should also be taken into consideration. (10)

The aim of this study was to analyze characteristics of patients with PE treated with thrombolytic therapy in our ICU, the bleeding or other complications of the therapy and the patient outcome.

\section{MATERIALS AND METHODS}

This is a retrospective analysis of patients with acute PE treated with recombinant tissue type plasminogen activator (tPA) alteplase in medical intensive care unit (ICU), University Hospital Sveti Duh. The study was conducted between March 2014 and April 2015. In that period 22 adult patients with PE received alteplase (Actylise, Boehringer Ingelheim Pharma $\mathrm{GmbH} \&$ Co.). In all patients the diagnosis was confirmed with CTPA. Written informed consent was obtained from all patients. Total dose of $100 \mathrm{mg}$ of alteplase was given via peripheral intravenous catheter as $2 \mathrm{~h}$ infusion. According to our protocol, which arises from previous vast experience with streptokinase, during alteplase infusion other anticoagulant agents were discontinued. After the end of alteplase, unfractionated heparin (UFH) was started in continuous infusion without the bolus dose. Further titration of UFH therapy was done on the basis of Raschke normogram. After a few days and clinical improvement, low molecular weight heparin (LMWH) was administered together with warfarin.

We reviewed medical charts on patients' characteristics (age, gender, symptoms of disease, risk factors), haemodynamic status (blood pressure and heart rate), CTPA finding and signs of right ventricular dysfunction (troponin I, echocardiography, electrocardiography). Pulmonary embolism severity index (PESI) and early mortality risk stratification was assessed for all patients. Evaluation of bleeding or other complications and patient outcome was done.

\section{RESULTS}

In the analysed period 75 patients $(10 \%$ of all hospitalized patients) with CTPA confirmed PE were hospitalized in the medical ICU. 22 (29\%) of them received thrombolytic therapy. Table 1 shows patients' characteristics. The mean age of patients treated with thrombolytic therapy was 63 years (range from 25 to 80 years), $45 \%$ were male, and $55 \%$ female. The major symptom in $73 \%$ of patients was dyspnoea, in $18 \%$ chest pain, and in $9 \%$ syncope. Two major risk factors for PE were malignancy (37\%) and immobilization (18\%). $27 \%$ of patients receiving alteplase had signs of hemodynamic instability while $73 \%$ were hemodynamically stable. All haemodynamically stable patients had echocardiographic signs of RV dysfunction. Considering ECG changes sinus tachycardia was most frequent ECG abnormality (64\%). Other abnormalities observed were: $\mathrm{T}$ wave inversion in V1-V3 (55\%), S1Q3T3 pattern (55\%), right bundle branch block (45\%). Only one patient had normal ECG. Troponin I was positive in all haemodynamically unstable patients, while in haemodynamically stable group it was positive in $50 \%$ of patients. Overall troponin I was positive in $58 \%$ of patients treated with thrombolytic therapy. All patients had an extensive clot burden on CTPA. Early mortality risk stratification was assessed for all patients. $27 \%$ were in the high risk group, $55 \%$ in the intermediate-high risk group, and $18 \%$ in the intermediate-low risk group. By using PESI criteria the majority of patients $(73 \%)$ were categorized in class $\mathrm{V}$ (very high mortality risk). Only one haemodynamically unstable patient (5\%) died, not because of PE or therapy complication, but from septic complication which occurred on the fourteenth day of hospitalization. All other patients survived and were discharged in improved condition. Two patients (9\%) had a major bleeding complication, which was successfully treated with supportive therapy with no need for invasive or surgical interventions. One patient from the intermediate-high risk group had skin and soft tissue bleeding and a second one who was haemodynamically unstable bled from puncture site of central vein catheter. Both patients recovered completely. There was no intracranial bleeding.
Table 1. Demographic and clinical patient data

\begin{tabular}{ll}
\hline $\mathbf{N}=\mathbf{2 2}$ & \\
\hline Age (mean) & $63 \pm 20,5$ \\
\hline Males/females & $10 / 12(45 / 55 \%)$ \\
\hline $\begin{array}{l}\text { Haemodynamically } \\
\text { unstable/stable }\end{array}$ & $6 / 16(27 / 73 \%)$ \\
\hline Symptoms & \\
\hline Dyspnea & $73 \%$ \\
\hline Chest pain & $18 \%$ \\
\hline Syncope & $9 \%$ \\
\hline
\end{tabular}

Early risk mortality

score

\begin{tabular}{ll}
\hline High & $27 \%$ \\
\hline Intermediate-high & $55 \%$ \\
\hline Intermediate-low & $18 \%$ \\
\hline low & $0 \%$ \\
\hline SPESI & \\
\hline Class I & $0 \%$ \\
\hline Class II & $18 \%$ \\
\hline Class III & $0 \%$ \\
\hline Class IV & $9 \%$ \\
\hline Class V & $73 \%$ \\
\hline
\end{tabular}

Echocardiographic signs 100\%

of RV dysphunction

\begin{tabular}{ll}
\hline Positive troponin I & $58 \%$ \\
\hline ECG abnormality & \\
\hline Tachycardia & $64 \%$ \\
\hline $\begin{array}{l}\text { T vawe inversion in } \\
\text { V1-V3 }\end{array}$ & $55 \%$ \\
\hline S1Q3T3 pattern & $55 \%$ \\
\hline $\begin{array}{l}\text { Right bundle branch } \\
\text { block }\end{array}$ & $45 \%$ \\
\hline Dead & $1(5 \%)$ \\
\hline
\end{tabular}

Bleeding complications $2(9 \%)$

RV - right ventricle, SPESI - simplified pulmonary embolism severity index 


\section{DISCUSSION}

Thrombolysis is an established treatment for patients with acute massive PE and haemodynamic instability. A review of randomized trials (12) and a recent epidemiological report (13) indicated that thrombolysis is associated with a reduction in mortality or recurrent PE in highrisk patients.

In contrast, the effect of thrombolytic therapy on the outcome of haemodynamically stable patients has been debated for many years and is still controversial. Situations in which clinicians contemplate thrombolytic therapy are: RV dysfunction, extensive clot burden, cardiopulmonal resuscitation, severe hypoxemia and free-floating right atrial or ventricular thrombus. In a randomized comparison of heparin vs. alteplase in 256 normotensive patients with acute PE and evidence of RV dysfunction or pulmonary hypertension, thrombolytic treatment improved the clinical course of patients and prevented further haemodynamic deterioration and the need for escalation to emergency treatment without affecting mortality. (10)

More recently, the Pulmonary Embolism Thrombolysis (PEITHO) trial was published that compared tenecteplase plus heparin with placebo plus heparin in 1006 patients with acute PE who were normotensive and had evidence of RV dysfunction. (14) The all-cause death or haemo- dynamic decompensation/collapse was significantly reduced with tenecteplase. In another randomized study comparing LMWH alone vs. LMWH plus an intravenous bolus of tenecteplase in intermediaterisk $\mathrm{PE}$, patients treated with tenecteplase had fewer adverse outcomes, better functional capacity, and greater quality of life. (15)

Thrombolytic treatment carries a risk of major bleeding, including intracranial haemorrhage. Analysis of pooled data from trials using various thrombolytic agents and regimens reported intracranial bleeding rates between $1.9 \%$ and $2.2 \%$. (11, 16) Increasing age and the presence of comorbidities have been associated with a higher risk of bleeding complications. (17) The PEITHO trial showed a $2 \%$ incidence of haemorrhagic stroke after thrombolytic treatment with tenecteplase (versus $0.2 \%$ in the placebo arm) in patients with intermediate-high-risk PE. (14)

The results from our study showed that thrombolytic therapy was frequently used in patients with acute PE. The majority of PE patients treated with thrombolytic therapy were haemodynamically stable and in the intermediate-high risk group. The decision to administer alteplase was conducted on a case-by-case basis after a thorough assessment of benefits and risks of thrombolytic therapy. Patient's preferences were also taken into consideration. Two major factors that contributed to the decision to administer alteplase were the echocardiographic signs of RV dysfunction and an extensive clot burden on CTPA as signs of potential haemodynamic destabilization. All haemodinamically stable patients survived and recovered completely. Overall none of the patients had intracranial bleeding, and only two patients had other bleeding complications, which were resolved completely without the need for surgical or invasive interventions. Although this study was done on a small population, we have positive experience with thrombolytic therapy in haemodynamically stable patients with PE with no intracranial bleeding complications so far. Further investigations on more patients are needed to determine exact recommendations for the use of thrombolytic therapy in haemodynamically stable patients with PE.

\section{CONCLUSION}

There are still controversies regarding the use of thrombolytic therapy in haemodynamically stable patients with PE. Treatment should be determined on a case-bycase basis after a thorough assessment of benefits and risks of thrombolytic therapy. When benefits outweigh the risks, thrombolytic therapy can be a life-saving procedure.

\section{REFERENCES}

1. Heit JA. The epidemiology of venous thromboembolism in the community. Arterioscler Thromb Vasc Biol 2008;28(3):370-372.

2. Cohen AT, Agnelli G, Anderson FA, Arcelus JI, Bergqvist D, Brecht JG, Greer IA, Heit JA, Hutchinson JL, Kakkar AK, Mottier D, Oger E, Samama MM, Spannagl M. Venous thromboembolism (VTE) in Europe. The number of VTE events and associated morbidity and mortality. Thromb Haemost 2007;98(4):756-764.

3. Heit JA III, Silverstein MD, Mohr DN, Petterson TM, O'Fallon WM, Melton LJ III. Risk factors for deep vein thrombosis and pulmonary embolism: a population based case-control study. Arch Intern Med 2000;160(6):809-815.

4. Pollack CV, Schreiber D, Goldhaber SZ, Slattery D, Fanikos J, O’Neil BJ, Thompson JR, Hiestand B, Briese BA, Pendleton RC, Miller $\mathrm{CD}$, Kline JA. Clinical characteristics, management, and outcomes of patients diagnosed with acute pulmonary embolism in the emergency department: initial report of EMPEROR(Multicenter Emergency Medicine PulmonaryEmbolism in the RealWorld Registry). J Am Coll Cardiol 2011;57(6):700-706. European Heart Journal (2014) 35, 3033-3080.

5. Konstantinides SV , Torbicki A, Agnelli G, Danchin N, Fitzmaurice D, Galiè N, Gibbs JS, Huisman MV, Humbert M, Kucher N, Lang I, Lankeit M, Lekakis J, Maack C, Mayer E, Meneveau N, Perrier A, Pruszczyk P, Rasmussen LH, Schindler TH, Svitil P, Vonk Noordegraaf A, Zamorano JL, Zompatori M. 2014 ESC Guidelines on the diagnosis and management of acute pulmonary embolism. Eur Heart J. 2014 Nov 14;35(43):3033-69.

6. Goldhaber SZ, Haire WD, Feldstein ML, Miller M, Toltzis R, Smith JL, Taveira da Silva AM,ComePC, Lee RT, Parker JA. Alteplase versus heparin in acute pulmonary embolism: randomised trial assessing right-ventricular function and pulmonary perfusion. Lancet 1993;341(8844):507-511.

7. Daniels LB, Parker JA, Patel SR, Grodstein F, Goldhaber SZ. Relation of duration of symptoms with response to thrombolytic therapy in pulmonary embolism. Am J Cardiol 1997;80(2):184-188.

8. Goldhaber SZ. Thrombolysis in pulmonary embolism: a debatable indication. Thromb Haemost 2001;86:444-451.

9. Goldhaber SZ. Thrombolysis in pulmonary embolism: a large-scale clinical trial is overdue. Circulation 2001;104:2876-2878 
10. Konstantinides S, Geibel A, Heusel G, Heinrich F, Kasper W. Heparin plus alteplase compared with heparin alone in patients with submassive pulmonary embolism. N Engl J Med. 2002 Oct 10;347(15):1143-50.

11. Kanter DS, Mikkola KM, Patel SR, Parker JA, Goldhaber SZ. Thrombolytic therapy for pulmonary embolism. Frequency of intracranial hemorrhage and associated risk factors. Chest 1997;111(5):1241-1245.

12. Wan S, Quinlan DJ, Agnelli G, EikelboomJW. Thrombolysiscompared with heparin for the initial treatment of pulmonary embolism: a meta-analysis of the randomized controlled trials. Circulation 2004;110(6):744-749.

13. Stein PD, Matta F. Thrombolytic therapy in unstable patients with acute pulmonary embolism: saves lives but underused. Am J Med 2012;125(5):465-470.

14. Meyer G, Vicaut E, DanaysT, Agnelli G, Becattini C, Beyer-Westendorf J, Bluhmki E, Bouvaist H, Brenner B, Couturaud F, Dellas C, Empen K, Franca A, Galie' N, Geibel A, Goldhaber SZ, Jimenez D, Kozak M, Kupatt C, Kucher N, Lang IM, Lankeit M, Meneveau N, Pacouret G, Palazzini M, Petris A, Pruszczyk P, Rugolotto M, Salvi A, Schellong S, Sebbane M, Sobkowicz B, Stefanovic BS, Thiele H, Torbicki A, Verschuren F, Konstantinides SV. Fibrinolysis for patients with intermediate-risk pulmonary embolism. N Engl J Med 2014;370(15):1402-1411.

15. Kline JA, Nordenholz KE, Courtney DM, Kabrhel C, Jones AE, Rondina MT, Diercks DB, Klinger JR, Hernandez J. Treatment of submassive pulmonary embolism with tenecteplase or placebo: cardiopulmonary outcomes at three months (TOPCOAT): Multicenter double-blind, placebo-controlled randomized trial. J Thromb Haemost 2014.

16. Levine MN, Goldhaber SZ, Gore JM, Hirsh J, Califf RM. Hemorrhagic complications of thrombolytic therapy in the treatment of myocardial infarction and venous thromboembolism. Chest 1995;108(4 Suppl):291S-301S.

17. Mikkola KM, Patel SR, Parker JA, Grodstein F, Goldhaber SZ. Increasing age is a major risk factor for hemorrhagic complications after pulmonary embolism thrombolysis. Am Heart J 1997;134(1):69-72. 